\title{
Uncertainty Principles for the Dunkl-Wigner Transforms
}

\author{
Fethi Soltani \\ Department of Mathematics, Faculty of Science, Jazan University, P.O. Box 277, Jazan 45142, Saudi Arabia
}

Correspondence should be addressed to Fethi Soltani; fethisoltani10@yahoo.com

Received 31 July 2016; Accepted 6 September 2016

Academic Editor: Dashan Fan

Copyright (C) 2016 Fethi Soltani. This is an open access article distributed under the Creative Commons Attribution License, which permits unrestricted use, distribution, and reproduction in any medium, provided the original work is properly cited.

We prove a version of Heisenberg-type uncertainty principle for the Dunkl-Wigner transform of magnitude $s>0$; and we deduce a local uncertainty principle for this transform.

\section{Introduction}

In this paper, we consider $\mathbb{R}^{d}$ with the Euclidean inner product $\langle\cdot, \cdot\rangle$ and norm $|y|:=\sqrt{\langle y, y\rangle}$. For $\alpha \in \mathbb{R}^{d} \backslash\{0\}$, let $\sigma_{\alpha}$ be the reflection in the hyperplane $H_{\alpha} \subset \mathbb{R}^{d}$ orthogonal to $\alpha$ :

$$
\sigma_{\alpha} y:=y-\frac{2\langle\alpha, y\rangle}{|\alpha|^{2}} \alpha .
$$

A finite set $\mathfrak{R} \subset \mathbb{R}^{d} \backslash\{0\}$ is called a root system, if $\Re \cap$ $\mathbb{R} . \alpha=\{-\alpha, \alpha\}$ and $\sigma_{\alpha} \mathfrak{R}=\mathfrak{R}$ for all $\alpha \in \mathfrak{R}$. We assume that it is normalized by $|\alpha|^{2}=2$ for all $\alpha \in \mathfrak{R}$. For a root system $\mathfrak{R}$, the reflections $\sigma_{\alpha}, \alpha \in \mathfrak{R}$, generate a finite group $G$. The Coxeter group $G$ is a subgroup of the orthogonal group $O(d)$. All reflections in $G$ correspond to suitable pairs of roots. For a given $\beta \in \mathbb{R}^{d} \backslash \bigcup_{\alpha \in \Re} H_{\alpha}$, we fix the positive subsystem $\mathfrak{R}_{+}:=\{\alpha \in \mathfrak{R}:\langle\alpha, \beta\rangle>0\}$. Then for each $\alpha \in \mathfrak{R}$ either $\alpha \in \mathfrak{R}_{+}$or $-\alpha \in \mathfrak{R}_{+}$.

Let $k: \mathfrak{R} \rightarrow \mathbb{C}$ be a multiplicity function on $\mathfrak{R}$ (a function which is constant on the orbits under the action of $G$ ). As an abbreviation, we introduce the index $\gamma=\gamma_{k}:=\sum_{\alpha \in \mathfrak{R}_{+}} k(\alpha)$.

Throughout this paper, we will assume that $k(\alpha) \geq 0$ for all $\alpha \in \mathfrak{R}$. Moreover, let $w_{k}$ denote the weight function $w_{k}(y):=\prod_{\alpha \in \Re_{+}}|\langle\alpha, y\rangle|^{2 k(\alpha)}$, for all $y \in \mathbb{R}^{d}$, which is Ginvariant and homogeneous of degree $2 \gamma$.

Let $c_{k}$ be the Mehta-type constant given by $c_{k}:=$ $\left(\int_{\mathbb{R}^{d}} e^{-|y|^{2} / 2} w_{k}(y) \mathrm{d} y\right)^{-1}$. We denote by $\mu_{k}$ the measure on $\mathbb{R}^{d}$ given by $\mathrm{d} \mu_{k}(y):=c_{k} w_{k}(y) \mathrm{d} y$, by $L^{p}\left(\mu_{k}\right), 1 \leq p \leq \infty$, the space of measurable functions $f$ on $\mathbb{R}^{d}$, such that

$$
\begin{aligned}
& \|f\|_{L^{p}\left(\mu_{k}\right)}:=\left(\int_{\mathbb{R}^{d}}|f(y)|^{p} \mathrm{~d} \mu_{k}(y)\right)^{1 / p}<\infty, \\
& \|f\|_{L^{\infty}\left(\mu_{k}\right)}:=\operatorname{ess} \sup _{y \in \mathbb{R}^{d}}|f(y)|<\infty,
\end{aligned}
$$

and by $L_{\mathrm{rad}}^{p}\left(\mu_{k}\right)$ the subspace of $L^{p}\left(\mu_{k}\right)$ consisting of radial functions. [1]) by

For $f \in L^{1}\left(\mu_{k}\right)$ the Dunkl transform of $f$ is defined (see

$$
\begin{aligned}
\mathscr{F}_{k}(f)(x):=\int_{\mathbb{R}^{d}} E_{k}(-i x, y) f(y) \mathrm{d} \mu_{k}(y), & \\
& x \in \mathbb{R}^{d},
\end{aligned}
$$

where $E_{k}(-i x, y)$ denotes the Dunkl kernel. (For more details see the next section.)

Many uncertainty principles have already been proved for the Dunkl transform $\mathscr{F}_{k}$, namely, by Rösler [2] and Shimeno [3] who established the Heisenberg-type uncertainty inequality for this transform, by showing that for $f \in L^{2}\left(\mu_{k}\right)$,

$$
\|f\|_{L^{2}\left(\mu_{k}\right)}^{2} \leq \frac{2}{2 \gamma+d}\||x| f\|_{L^{2}\left(\mu_{k}\right)}\left\||y| \mathscr{F}_{k}(f)\right\|_{L^{2}\left(\mu_{k}\right)} .
$$


Recently, the author [4-7] proved general forms of the Heisenberg-type inequality for the Dunkl transform $\mathscr{F}_{k}$.

The Dunkl translation operators $\tau_{x}, x \in \mathbb{R}^{d}$, [8] are defined on $L^{2}\left(\mu_{k}\right)$ by

$$
\mathscr{F}_{k}\left(\tau_{x} f\right)(y)=E_{k}(i x, y) \mathscr{F}_{k}(f)(y), \quad y \in \mathbb{R}^{d} .
$$

Let $g \in L_{\text {rad }}^{2}\left(\mu_{k}\right)$. The Dunkl-Wigner transform $V_{g}$ is the mapping defined for $f \in L^{2}\left(\mu_{k}\right)$ by

$$
V_{g}(f)(x, y):=\int_{\mathbb{R}^{d}} f(t) \overline{\tau_{x} g_{k, y}(-t)} \mathrm{d} \mu_{k}(t),
$$

where

$$
g_{k, y}(z):=\mathscr{F}_{k}\left(\sqrt{\tau_{y}\left|\mathscr{F}_{k}(g)\right|^{2}}\right)(z)
$$

This transform is studied in $[9,10]$ where the author established some applications (Plancherel formula, inversion formula, Calderón's reproducing formula, extremal function, etc.).

In this paper we use formula (4); we prove uncertainty principle intervening $\mathscr{F}_{k}$ and $V_{g}$ of magnitudes $a, b \geq 1$; that is, for every $f \in L^{2}\left(\mu_{k}\right)$,

$$
\begin{gathered}
\left\||x|^{a} V_{g}(f)\right\|_{L^{2}\left(\mu_{k} \otimes \mu_{k}\right)}^{b}\left\||z|^{b} \mathscr{F}_{k}(f)\right\|_{L^{2}\left(\mu_{k}\right)}^{a} \\
\geq\left(\gamma+\frac{d}{2}\right)^{a b}\|f\|_{L^{2}\left(\mu_{k}\right)}^{a+b}\|g\|_{L_{\mathrm{rad}}^{2}\left(\mu_{k}\right)}^{b} .
\end{gathered}
$$

Next, we prove a Heisenberg-type uncertainty principle for the Dunkl-Wigner transform $V_{g}$ of magnitude $s>0$; that is, there exists a constant $c(k, s)>0$ such that, for $f \in L^{2}\left(\mu_{k}\right)$,

$$
\begin{aligned}
& \left\||x|^{s} V_{g}(f)\right\|_{L^{2}\left(\mu_{k} \otimes \mu_{k}\right)}\left\||y|^{s} V_{g}(f)\right\|_{L^{2}\left(\mu_{k} \otimes \mu_{k}\right)} \\
& \geq c(k, s)\|f\|_{L^{2}\left(\mu_{k}\right)}^{2}\|g\|_{L_{\mathrm{rad}}^{2}\left(\mu_{k}\right)}^{2} .
\end{aligned}
$$

Finally, we prove a local uncertainty principle for the DunklWigner transform $V_{g}$; that is, there exists a constant $b(k, s)>$ 0 such that, for $f \in L^{2}\left(\mu_{k}\right)$ and for measurable subset $E$ of $\mathbb{R}^{d} \times \mathbb{R}^{d}$ such that $0<\mu_{k} \otimes \mu_{k}(E)<\infty$,

$$
\begin{aligned}
& \left\|\chi_{E} V_{g}(f)\right\|_{L^{2}\left(\mu_{k} \otimes \mu_{k}\right)} \\
& \quad \leq b(k, s)\left(\mu_{k} \otimes \mu_{k}(E)\right)^{1 / 2}\left\||(x, y)|^{s} V_{g}(f)\right\|_{L^{2}\left(\mu_{k} \otimes \mu_{k}\right)},
\end{aligned}
$$

where $\chi_{E}$ is the indicator function of the set $E$.

In the classical case, the Fourier-Wigner transforms are studied by Weyl [11] and Wong [12]. In the Bessel-Kingman hypergroups, these operators are studied by Dachraoui [13].

This paper is organized as follows. In Section 2, we recall some properties of the Dunkl-Wigner transform $V_{g}$. In Section 3, we prove a Heisenberg-type uncertainty principle for the Dunkl-Wigner transform $V_{g}$ of magnitude $s>0$; and we deduce a local uncertainty principle for this transform.

\section{The Dunkl-Wigner Transform}

The Dunkl operators $\mathscr{D}_{j}, j=1, \ldots, d$, on $\mathbb{R}^{d}$ associated with the finite reflection group $G$ and multiplicity function $k$ are given, for a function $f$ of class $C^{1}$ on $\mathbb{R}^{d}$, by

$$
\mathscr{D}_{j} f(y):=\frac{\partial}{\partial y_{j}} f(y)+\sum_{\alpha \in \Re_{+}} k(\alpha) \alpha_{j} \frac{f(y)-f\left(\sigma_{\alpha} y\right)}{\langle\alpha, y\rangle} .
$$

For $y \in \mathbb{R}^{d}$, the initial value problem $\mathscr{D}_{j} u(\cdot, y)(x)=$ $y_{j} u(x, y), j=1, \ldots, d$, with $u(0, y)=1$ admits a unique analytic solution on $\mathbb{R}^{d}$, which will be denoted by $E_{k}(x, y)$ and called Dunkl kernel $[14,15]$. This kernel has a unique analytic extension to $\mathbb{C}^{d} \times \mathbb{C}^{d}$ (see [16]). The Dunkl kernel has the Laplace-type representation [17]

$$
E_{k}(x, y)=\int_{\mathbb{R}^{d}} e^{\langle y, z\rangle} \mathrm{d} \Gamma_{x}(z), \quad x \in \mathbb{R}^{d}, y \in \mathbb{C}^{d}
$$

where $\langle y, z\rangle:=\sum_{i=1}^{d} y_{i} z_{i}$ and $\Gamma_{x}$ is a probability measure on $\mathbb{R}^{d}$, such that $\operatorname{supp}\left(\Gamma_{x}\right) \subset\left\{z \in \mathbb{R}^{d}:|z| \leq|x|\right\}$. In our case,

$$
\left|E_{k}( \pm i x, y)\right| \leq 1, \quad x, y \in \mathbb{R}^{d} .
$$

The Dunkl kernel gives rise to an integral transform, which is called Dunkl transform on $\mathbb{R}^{d}$, and was introduced by Dunkl in [1], where already many basic properties were established. Dunkl's results were completed and extended later by de Jeu [15]. The Dunkl transform of a function $f$ in $L^{1}\left(\mu_{k}\right)$ is defined by

$$
\begin{aligned}
\mathscr{F}_{k}(f)(x):=\int_{\mathbb{R}^{d}} E_{k}(-i x, y) f(y) \mathrm{d} \mu_{k}(y), & \\
& x \in \mathbb{R}^{d} .
\end{aligned}
$$

We notice that $\mathscr{F}_{0}$ agrees with the Fourier transform $\mathscr{F}$ that is given by

$$
\mathscr{F}(f)(x):=(2 \pi)^{-d / 2} \int_{\mathbb{R}^{d}} e^{-i\langle x, y\rangle} f(y) \mathrm{d} y, \quad x \in \mathbb{R}^{d} .
$$

The Dunkl transform of a function $f \in L_{\mathrm{rad}}^{1}\left(\mu_{k}\right)$ which is radial is again radial and could be computed via the associated Fourier-Bessel transform $\mathscr{F}_{\gamma+d / 2-1}^{B}$ (see [18], Proposition 4); that is,

$$
\mathscr{F}_{k}(f)(x)=\mathscr{F}_{\gamma+d / 2-1}^{B}(F)(|x|),
$$

where $f(x)=F(|x|)$ and

$$
\begin{aligned}
& \mathscr{F}_{\gamma+d / 2-1}^{B}(F)(|x|) \\
& \quad:=\int_{0}^{\infty} F(r) \frac{j_{\gamma+d / 2-1}(|x| r)}{2^{\gamma+d / 2-1} \Gamma(\gamma+d / 2)} r^{2 \gamma+d-1} \mathrm{~d} r .
\end{aligned}
$$

Here $j_{\gamma}$ is the spherical Bessel function (see [19]).

Some of the properties of Dunkl transform $\mathscr{F}_{k}$ are collected below (see $[1,15])$. 
Theorem 1. (i) $L^{1}-L^{\infty}$-Boundedness. For all $f \in L^{1}\left(\mu_{k}\right)$, $\mathscr{F}_{k}(f) \in L^{\infty}\left(\mu_{k}\right)$, and

$$
\left\|\mathscr{F}_{k}(f)\right\|_{L^{\infty}\left(\mu_{k}\right)} \leq\|f\|_{L^{1}\left(\mu_{k}\right)} .
$$

(ii) Inversion Theorem. Let $f \in L^{1}\left(\mu_{k}\right)$, such that $\mathscr{F}_{k}(f) \in$ $L^{1}\left(\mu_{k}\right)$. Then

$$
f(x)=\mathscr{F}_{k}\left(\mathscr{F}_{k}(f)\right)(-x) \text {, a.e. } x \in \mathbb{R}^{d} .
$$

(iii) Plancherel Theorem. The Dunkl transform $\mathscr{F}_{k}$ extends uniquely to an isometric isomorphism of $L^{2}\left(\mu_{k}\right)$ onto itself. In particular, one has

$$
\|f\|_{L^{2}\left(\mu_{k}\right)}=\left\|\mathscr{F}_{k}(f)\right\|_{L^{2}\left(\mu_{k}\right)} .
$$

(iv) Parseval Theorem. For $f, g \in L^{2}\left(\mu_{k}\right)$, one has

$$
\langle f, g\rangle_{L^{2}\left(\mu_{k}\right)}=\left\langle\mathscr{F}_{k}(f), \mathscr{F}_{k}(g)\right\rangle_{L^{2}\left(\mu_{k}\right)} .
$$

The Dunkl transform $\mathscr{F}_{k}$ allows us to define a generalized translation operators on $L^{2}\left(\mu_{k}\right)$ by setting

$$
\mathscr{F}_{k}\left(\tau_{x} f\right)(y)=E_{k}(i x, y) \mathscr{F}_{k}(f)(y), \quad y \in \mathbb{R}^{d} .
$$

It is the definition of Thangavelu and Xu given in [8]. It plays the role of the ordinary translation $\tau_{x} f=f(x+\cdot)$ in $\mathbb{R}^{d}$, since the Euclidean Fourier transform satisfies $\mathscr{F}\left(\tau_{x} f\right)(y)=$ $e^{i\langle x, y\rangle} \mathscr{F}(f)(y)$. Note that, from (13) and Theorem 1(iii), relation (22) makes sense, and $\left\|\tau_{x} f\right\|_{L^{2}\left(\mu_{k}\right)} \leq\|f\|_{L^{2}\left(\mu_{k}\right)}$, for all $f \in L^{2}\left(\mu_{k}\right)$.

Rösler [20] introduced the Dunkl translation operators for radial functions. If $f$ are radial functions, $f(x)=F(|x|)$, then

$$
\begin{array}{r}
\tau_{x} f(y)=\int_{\mathbb{R}^{d}} F\left(\sqrt{|x|^{2}+|y|^{2}+2\langle y, z\rangle}\right) \mathrm{d} \Gamma_{x}(z) ; \\
x, y \in \mathbb{R}^{d},
\end{array}
$$

where $\Gamma_{x}$ is the representing measure given by (12). $[8,21]$

This formula allows us to establish the following results

Proposition 2. (i) For all $p \in[1,2]$ and for all $x \in \mathbb{R}^{d}$, the Dunkl translation $\tau_{x}: L_{r a d}^{p}\left(\mu_{k}\right) \rightarrow L^{p}\left(\mu_{k}\right)$ is a bounded operator, and for $f \in L_{\text {rad }}^{p}\left(\mu_{k}\right)$, one has

$$
\left\|\tau_{x} f\right\|_{L^{p}\left(\mu_{k}\right)} \leq\|f\|_{L_{r a d}^{p}\left(\mu_{k}\right)} .
$$

(ii) Let $f \in L_{\text {rad }}^{1}\left(\mu_{k}\right)$. Then, for all $x \in \mathbb{R}^{d}$, one has

$$
\int_{\mathbb{R}^{d}} \tau_{x} f(y) \mathrm{d} \mu_{k}(y)=\int_{\mathbb{R}^{d}} f(y) \mathrm{d} \mu_{k}(y) .
$$

The Dunkl convolution product $*_{k}$ of two functions $f$ and $g$ in $L^{2}\left(\mu_{k}\right)$ is defined by

$$
f *{ }_{k} g(x):=\int_{\mathbb{R}^{d}} \tau_{x} f(-y) g(y) \mathrm{d} \mu_{k}(y), \quad x \in \mathbb{R}^{d} .
$$

We notice that $*_{k}$ generalizes the convolution $*$ that is given by

$$
f * g(x):=(2 \pi)^{-d / 2} \int_{\mathbb{R}^{d}} f(x-y) g(y) \mathrm{d} y,
$$

$$
x \in \mathbb{R}^{d} .
$$

Proposition 2 allows us to establish the following properties for the Dunkl convolution on $\mathbb{R}^{d}$ (see [8]).

Proposition 3. (i) Assume that $p \in[1,2]$ and $q, r \in[1, \infty]$ such that $1 / p+1 / q=1+1 / r$. Then the $\operatorname{map}(f, g) \rightarrow f{ }_{k} g$ extends to a continuous map from $L_{\text {rad }}^{p}\left(\mu_{k}\right) \times L^{q}\left(\mu_{k}\right)$ to $L^{r}\left(\mu_{k}\right)$, and

$$
\left\|f *_{k} g\right\|_{L^{r}\left(\mu_{k}\right)} \leq\|f\|_{L_{r a d}^{p}\left(\mu_{k}\right)}\|g\|_{L^{q}\left(\mu_{k}\right)} .
$$

(ii) For all $f \in L_{\text {rad }}^{1}\left(\mu_{k}\right)$ and $g \in L^{2}\left(\mu_{k}\right)$, one has

$$
\mathscr{F}_{k}\left(f *_{k} g\right)=\mathscr{F}_{k}(f) \mathscr{F}_{k}(g) \text {. }
$$

(iii) Let $f \in L_{\text {rad }}^{2}\left(\mu_{k}\right)$ and $g \in L^{2}\left(\mu_{k}\right)$. Then $f *_{k} g$ belongs to $L^{2}\left(\mu_{k}\right)$ if and only if $\mathscr{F}_{k}(f) \mathscr{F}_{k}(g)$ belongs to $L^{2}\left(\mu_{k}\right)$, and

$$
\begin{aligned}
& \mathscr{F}_{k}\left(f *_{k} g\right)=\mathscr{F}_{k}(f) \mathscr{F}_{k}(g), \\
& \text { in the } L^{2}\left(\mu_{k}\right) \text {-case. }
\end{aligned}
$$

(iv) Let $f \in L_{\text {rad }}^{2}\left(\mu_{k}\right)$ and $g \in L^{2}\left(\mu_{k}\right)$. Then

$$
\begin{aligned}
& \int_{\mathbb{R}^{d}}|f * g(x)|^{2} \mathrm{~d} \mu_{k}(x) \\
& \quad=\int_{\mathbb{R}^{d}}\left|\mathscr{F}_{k}(f)(z)\right|^{2}\left|\mathscr{F}_{k}(g)(z)\right|^{2} \mathrm{~d} \mu_{k}(z),
\end{aligned}
$$

where both sides are finite or infinite.

Let $g \in L_{\text {rad }}^{2}\left(\mu_{k}\right)$ and $y \in \mathbb{R}^{d}$. The modulation of $g$ by $y$ is the function $g_{k, y}$ defined by

$$
g_{k, y}(z):=\mathscr{F}_{k}\left(\sqrt{\tau_{y}\left|\mathscr{F}_{k}(g)\right|^{2}}\right)(z), \quad z \in \mathbb{R}^{d} .
$$

Thus,

$$
\left\|g_{k, y}\right\|_{L^{2}\left(\mu_{k}\right)}=\|g\|_{L_{\text {rad }}^{2}\left(\mu_{k}\right)} .
$$

Let $g \in L_{\text {rad }}^{2}\left(\mu_{k}\right)$. The Fourier-Wigner transform associated with the Dunkl operators is the mapping $V_{g}$ defined for $f \in L^{2}\left(\mu_{k}\right)$ by

$$
\begin{array}{r}
V_{g}(f)(x, y):=\int_{\mathbb{R}^{d}} f(t) \overline{\tau_{x} g_{k, y}(-t)} \mathrm{d} \mu_{k}(t), \\
x, y \in \mathbb{R}^{d} .
\end{array}
$$

In the following we recall some properties of the DunklWigner transform (Plancherel formula, inversion formula, reproducing inversion formula of Calderón's type, etc.). 
Proposition 4 (see [10]). Let $(f, g) \in L^{2}\left(\mu_{k}\right) \times L_{\text {rad }}^{2}\left(\mu_{k}\right)$. Then

(i) $V_{g}(f)(x, y)=\overline{g_{k, y}} *_{k} f(x)$.

(ii) $V_{g}(f)(x, y)=\int_{\mathbb{R}^{d}} E_{k}(i x, z) \mathscr{F}_{k}(f)(z) \sqrt{\tau_{y}\left|\mathscr{F}_{k}(g)\right|^{2}(z)}$ $\mathrm{d} \mu_{k}(z)$.

(iii) The function $V_{g}(f)$ belongs to $L^{\infty}\left(\mu_{k} \otimes \mu_{k}\right)$, and

$$
\left\|V_{g}(f)\right\|_{L^{\infty}\left(\mu_{k} \otimes \mu_{k}\right)} \leq\|f\|_{L^{2}\left(\mu_{k}\right)}\|g\|_{L_{r a d}^{2}\left(\mu_{k}\right)} .
$$

Theorem 5 (see [10]). Let $g \in L_{\text {rad }}^{2}\left(\mu_{k}\right)$ be a nonzero function. Then one has the following.

(i) Plancherel formula: for every $f \in L^{2}\left(\mu_{k}\right)$, one has

$$
\left\|V_{g}(f)\right\|_{L^{2}\left(\mu_{k} \otimes \mu_{k}\right)}=\|f\|_{L^{2}\left(\mu_{k}\right)}\|g\|_{L_{r a d}^{2}\left(\mu_{k}\right)} .
$$

(ii) Parseval formula: for every $f, h \in L^{2}\left(\mu_{k}\right)$, one has

$$
\left\langle V_{g}(f), V_{g}(h)\right\rangle_{L^{2}\left(\mu_{k} \otimes \mu_{k}\right)}=\|g\|_{L_{r a d}^{2}\left(\mu_{k}\right)}^{2}\langle f, h\rangle_{L^{2}\left(\mu_{k}\right)} .
$$

(iii) Inversion formula: for all $f \in L^{1} \cap L^{2}\left(\mu_{k}\right)$ such that $\mathscr{F}_{k}(f) \in L^{1}\left(\mu_{k}\right)$, one has

$$
\begin{aligned}
& f(z)=\frac{1}{\|g\|_{L_{r a d}^{2}\left(\mu_{k}\right)}^{2}} \\
& \quad \cdot \iint_{\mathbb{R}^{d}} V_{g}(f)(x, y) \overline{\tau_{z} g_{k, y}(-x)} \mathrm{d} \mu_{k}(x) \mathrm{d} \mu_{k}(y) .
\end{aligned}
$$

Theorem 6 (Calderón's reproducing inversion formula; see [10]). Let $\Delta=\prod_{j=1}^{d}\left[a_{j}, b_{j}\right],-\infty<a_{j}<b_{j}<\infty$, and let $g \in$ $L_{\text {rad }}^{2}\left(\mu_{k}\right)$ be a nonzero function, such that $\mathscr{F}_{k}(g) \in L^{\infty}\left(\mu_{k}\right)$. Then, for $f \in L^{2}\left(\mu_{k}\right)$, the function $f_{\Delta}$ given by

$$
\begin{aligned}
& f_{\Delta}(z)=\frac{1}{\|g\|_{L_{\text {rad }}^{2}\left(\mu_{k}\right)}} \\
& \quad \cdot \int_{\Delta} \int_{\mathbb{R}^{d}} V_{g}(f)(x, y) \overline{\tau_{z} g_{k, y}(-x)} \mathrm{d} \mu_{k}(x) \mathrm{d} \mu_{k}(y)
\end{aligned}
$$

belongs to $L^{2}\left(\mu_{k}\right)$ and satisfies

$$
\lim _{\substack{a_{j} \rightarrow-\infty \\ b_{j} \rightarrow+\infty}}\left\|f_{\Delta}-f\right\|_{L^{2}\left(\mu_{k}\right)}=0 .
$$

\section{Uncertainty Principles for the Mapping $V_{g}$}

In this section we establish Heisenberg-type uncertainty principle for the Dunkl-Wigner transform $V_{g}$. We begin by the following theorem.

Theorem 7. Let $g \in L_{\text {rad }}^{2}\left(\mu_{k}\right)$ be a nonzero function. Then, for $f \in L^{2}\left(\mu_{k}\right)$, one has

$$
\begin{gathered}
\left\||x| V_{g}(f)\right\|_{L^{2}\left(\mu_{k} \otimes \mu_{k}\right)}\left\||z| \mathscr{F}_{k}(f)\right\|_{L^{2}\left(\mu_{k}\right)} \\
\geq\left(\gamma+\frac{d}{2}\right)\|f\|_{L^{2}\left(\mu_{k}\right)}^{2}\|g\|_{L_{r a d}^{2}\left(\mu_{k}\right)} .
\end{gathered}
$$

Proof. Let $f \in L^{2}\left(\mu_{k}\right)$. Assume that $\left\||x| V_{g}(f)\right\|_{L^{2}\left(\mu_{k} \otimes \mu_{k}\right)}+$ $\left\||z| \mathscr{F}_{k}(f)\right\|_{L^{2}\left(\mu_{k}\right)}<\infty$. Inequality (4) leads to

$$
\begin{aligned}
& \int_{\mathbb{R}^{d}}\left|V_{g}(f)(x, y)\right|^{2} \mathrm{~d} \mu_{k}(x) \\
& \leq \frac{2}{2 \gamma+d}\left(\int_{\mathbb{R}^{d}}|x|^{2}\left|V_{g}(f)(x, y)\right|^{2} \mathrm{~d} \mu_{k}(x)\right)^{1 / 2} \\
& \left(\int_{\mathbb{R}^{d}}|z|^{2}\left|\mathscr{F}_{k}\left(V_{g}(f)(\cdot, y)\right)(z)\right|^{2} \mathrm{~d} \mu_{k}(z)\right)^{1 / 2} .
\end{aligned}
$$

Integrating with respect to $\mathrm{d} \mu_{k}(y)$ and using the Schwarz inequality, we get

$$
\begin{aligned}
& \left.\left.\cdot(x, y)\right|^{2} \mathrm{~d} \mu_{k}(x) \mathrm{d} \mu_{k}(y)\right)^{1 / 2} \\
& \cdot\left(\iint_{\mathbb{R}^{d}}|z|^{2} \mid \mathscr{F}_{k}\left(V_{g}(f)(\cdot, y)\right)\right. \\
& \left.\left.\cdot(z)\right|^{2} \mathrm{~d} \mu_{k}(z) \mathrm{d} \mu_{k}(y)\right)^{1 / 2} .
\end{aligned}
$$$$
\left\|V_{g}(f)\right\|_{L^{2}\left(\mu_{k} \otimes \mu_{k}\right)}^{2} \leq \frac{2}{2 \gamma+d}\left(\iint_{\mathbb{R}^{d}}|x|^{2} \mid V_{g}(f)\right.
$$

But by Proposition 4(ii), Fubini-Tonelli's theorem, (16), Proposition 2(ii), and Theorem 1(iii), we have

$$
\begin{aligned}
& \iint_{\mathbb{R}^{d}}|z|^{2}\left|\mathscr{F}_{k}\left(V_{g}(f)(\cdot, y)\right)(z)\right|^{2} \mathrm{~d} \mu_{k}(z) \mathrm{d} \mu_{k}(y) \\
& \quad=\iint_{\mathbb{R}^{d}}|z|^{2} \tau_{y}\left|\mathscr{F}_{k}(g)\right|^{2}(z) \mid \mathscr{F}_{k}(f) \\
& \left.\quad \cdot(z)\right|^{2} \mathrm{~d} \mu_{k}(z) \mathrm{d} \mu_{k}(y) \\
& \quad=\|g\|_{L_{\text {rad }}^{2}\left(\mu_{k}\right)}^{2} \int_{\mathbb{R}^{d}}|z|^{2}\left|\mathscr{F}_{k}(f)(z)\right|^{2} \mathrm{~d} \mu_{k}(z) .
\end{aligned}
$$

This yields the result and completes the proof of the theorem.

Theorem 8. Let $g \in L_{\text {rad }}^{2}\left(\mu_{k}\right)$ be a nonzero function and $s \geq 1$. Then, for $f \in L^{2}\left(\mu_{k}\right)$, one has

$$
\begin{gathered}
\left\||x|^{s} V_{g}(f)\right\|_{L^{2}\left(\mu_{k} \otimes \mu_{k}\right)}\left\||z|^{s} \mathscr{F}_{k}(f)\right\|_{L^{2}\left(\mu_{k}\right)} \\
\geq\left(\gamma+\frac{d}{2}\right)^{s}\|f\|_{L^{2}\left(\mu_{k}\right)}^{2}\|g\|_{L_{r a d}^{2}\left(\mu_{k}\right)} .
\end{gathered}
$$

Proof. Let $s \geq 1$ and let $f \in L^{2}\left(\mu_{k}\right), f \neq 0$, such that $\left\||x|^{s} V_{g}(f)\right\|_{L^{2}\left(\mu_{k} \otimes \mu_{k}\right)}+\left\||z|^{s} \mathscr{F}_{k}(f)\right\|_{L^{2}\left(\mu_{k}\right)}<\infty$. Then, for $s>1$, we have

$$
\begin{aligned}
& \left\||x|^{s} V_{g}(f)\right\|_{L^{2}\left(\mu_{k} \otimes \mu_{k}\right)}^{2 / s}\left\|V_{g}(f)\right\|_{L^{2}\left(\mu_{k} \otimes \mu_{k}\right)}^{2 / s^{\prime}} \\
& =\left\||x|^{2}\left|V_{g}(f)\right|^{2 / s}\right\|_{L^{s}\left(\mu_{k} \otimes \mu_{k}\right)}\left\|\left|V_{g}(f)\right|^{2 / s^{\prime}}\right\|_{L^{s^{\prime}}\left(\mu_{k} \otimes \mu_{k}\right)},
\end{aligned}
$$


where $s^{\prime}$ is defined as usual by $1 / s+1 / s^{\prime}=1$. By Hölder's inequality we get

$$
\begin{aligned}
& \left\||x|^{s} V_{g}(f)\right\|_{L^{2}\left(\mu_{k} \otimes \mu_{k}\right)}^{1 / s}\left\|V_{g}(f)\right\|_{L^{2}\left(\mu_{k} \otimes \mu_{k}\right)}^{1 / s^{\prime}} \\
& \quad \geq\left\||x| V_{g}(f)\right\|_{L^{2}\left(\mu_{k} \otimes \mu_{k}\right)} .
\end{aligned}
$$

Thus, for all $s \geq 1$, we have

$$
\left\||x|^{s} V_{g}(f)\right\|_{L^{2}\left(\mu_{k} \otimes \mu_{k}\right)}^{1 / s} \geq \frac{\left\||x| V_{g}(f)\right\|_{L^{2}\left(\mu_{k} \otimes \mu_{k}\right)}}{\left\|V_{g}(f)\right\|_{L^{2}\left(\mu_{k} \otimes \mu_{k}\right)}^{1-1 /}},
$$

with equality if $s=1$. In the same manner and using Theorem 1(iii), we have, for $s \geq 1$,

$$
\left\||z|^{s} \mathscr{F}_{k}(f)\right\|_{L^{2}\left(\mu_{k}\right)}^{1 / s} \geq \frac{\left\||z| \mathscr{F}_{k}(f)\right\|_{L^{2}\left(\mu_{k}\right)}}{\left\|\mathscr{F}_{k}(f)\right\|_{L^{2}\left(\mu_{k}\right)}^{1-1 / s}},
$$

with equality if $s=1$. By (48) and (49), for all $s \geq 1$, we have

$$
\begin{aligned}
& \left\||x|^{s} V_{g}(f)\right\|_{L^{2}\left(\mu_{k} \otimes \mu_{k}\right)}^{1 / s}\left\||z|^{s} \mathscr{F}_{k}(f)\right\|_{L^{2}\left(\mu_{k}\right)}^{1 / s} \\
& \geq \frac{\left\||x| V_{g}(f)\right\|_{L^{2}\left(\mu_{k} \otimes \mu_{k}\right)}\left\||z| \mathscr{F}_{k}(f)\right\|_{L^{2}\left(\mu_{k}\right)}}{\left(\|f\|_{L^{2}\left(\mu_{k}\right)}^{2}\|g\|_{L_{\text {rad }}^{2}\left(\mu_{k}\right)}\right)^{1-1 / s}},
\end{aligned}
$$

with equality if $s=1$. Applying Theorem 7 , we obtain

$$
\begin{gathered}
\left\||x|^{s} V_{g}(f)\right\|_{L^{2}\left(\mu_{k} \otimes \mu_{k}\right)}\left\||z|^{s} \mathscr{F}_{k}(f)\right\|_{L^{2}\left(\mu_{k}\right)} \\
\geq\left(\gamma+\frac{d}{2}\right)^{s}\|f\|_{L^{2}\left(\mu_{k}\right)}^{2}\|g\|_{L_{\text {rad }}^{2}\left(\mu_{k}\right)},
\end{gathered}
$$

which completes the proof of the theorem.

From (48) and (49) we deduce the following remark.

Remark 9. Let $g \in L_{\text {rad }}^{2}\left(\mu_{k}\right)$ be a nonzero function and $a, b \geq$ 1. Then, for $f \in L^{2}\left(\mu_{k}\right)$, we have

$$
\begin{gathered}
\left\||x|^{a} V_{g}(f)\right\|_{L^{2}\left(\mu_{k} \otimes \mu_{k}\right)}^{b}\left\||z|^{b} \mathscr{F}_{k}(f)\right\|_{L^{2}\left(\mu_{k}\right)}^{a} \\
\geq\left(\gamma+\frac{d}{2}\right)^{a b}\|f\|_{L^{2}\left(\mu_{k}\right)}^{a+b}\|g\|_{L_{\text {rad }}^{2}\left(\mu_{k}\right)}^{b} .
\end{gathered}
$$

For $\lambda>0$, we define the dilation of $f \in L^{2}\left(\mu_{k}\right)$ by

$$
f_{\lambda}(x):=\frac{1}{\lambda^{\gamma+d / 2}} f\left(\frac{x}{\lambda}\right), \quad x \in \mathbb{R}^{d} .
$$

Then

$$
\begin{aligned}
\left\|f_{\lambda}\right\|_{L^{2}\left(\mu_{k}\right)} & =\|f\|_{L^{2}\left(\mu_{k}\right)}, \\
\mathscr{F}_{k}\left(f_{\lambda}\right)(z) & =\lambda^{\gamma+d / 2} \mathscr{F}_{k}(f)(\lambda z), \\
\tau_{x}\left(f_{\lambda}\right)(y) & =\frac{1}{\lambda^{\gamma+d / 2}} \tau_{x / \lambda} f\left(\frac{y}{\lambda}\right) .
\end{aligned}
$$

Let us now turn to establishing Heisenberg-type uncertainty principle for the Dunkl-Wigner transform $V_{g}$ of magnitude $s>0$. Thus, we consider the following lemma.
Lemma 10. Let $\lambda>0$ and let $g \in L_{\text {rad }}^{2}\left(\mu_{k}\right)$ be a nonzero function. Then, for $f \in L^{2}\left(\mu_{k}\right)$, one has

$$
V_{g_{\lambda}}\left(f_{\lambda}\right)(x, y)=V_{g}(f)\left(\frac{x}{\lambda}, \lambda y\right), \quad x, y \in \mathbb{R}^{d} .
$$

Proof. From Proposition 4(ii), we have

$$
\begin{aligned}
& V_{g_{\lambda}}\left(f_{\lambda}\right)(x, y)=\int_{\mathbb{R}^{d}} E_{k}(i x, z) \mathscr{F}_{k}\left(f_{\lambda}\right)(z) \\
& \cdot \sqrt{\tau_{y}\left|\mathscr{F}_{k}\left(g_{\lambda}\right)\right|^{2}(z)} \mathrm{d} \mu_{k}(z) .
\end{aligned}
$$

But by (55) we have

$$
\tau_{y}\left|\mathscr{F}_{k}\left(g_{\lambda}\right)\right|^{2}(z)=\lambda^{2 \gamma+d} \tau_{\lambda y}\left|\mathscr{F}_{k}(g)\right|^{2}(\lambda z) .
$$

Thus,

$$
\begin{aligned}
& V_{g_{\lambda}}\left(f_{\lambda}\right)(x, y)=\lambda^{2 \gamma+d} \int_{\mathbb{R}^{d}} E_{k}(i x, z) \mathscr{F}_{k}(f)(\lambda z) \\
& \cdot \sqrt{\tau_{\lambda y}\left|\mathscr{F}_{k}(g)\right|^{2}(\lambda z)} \mathrm{d} \mu_{k}(z)=\int_{\mathbb{R}^{d}} E_{k}\left(i x, \frac{z}{\lambda}\right) \\
& \cdot \mathscr{F}_{k}(f)(z) \sqrt{\tau_{\lambda y}\left|\mathscr{F}_{k}(g)\right|^{2}(z)} \mathrm{d} \mu_{k}(z)=V_{g}(f) \\
& \cdot\left(\frac{x}{\lambda}, \lambda y\right),
\end{aligned}
$$

which gives the result.

Theorem 11 (Heisenberg-type uncertainty principle for $V_{g}$ ). Let $s>0$. Then there exists a constant $c(k, s)>0$ such that, for all $f \in L^{2}\left(\mu_{k}\right)$ and $g \in L_{\text {rad }}^{2}\left(\mu_{k}\right)$, one has

$$
\begin{gathered}
\left\||x|^{s} V_{g}(f)\right\|_{L^{2}\left(\mu_{k} \otimes \mu_{k}\right)}\left\||y|^{s} V_{g}(f)\right\|_{L^{2}\left(\mu_{k} \otimes \mu_{k}\right)} \\
\geq c(k, s)\|f\|_{L^{2}\left(\mu_{k}\right)}^{2}\|g\|_{L_{\text {rad }}^{2}\left(\mu_{k}\right)}^{2} .
\end{gathered}
$$

Proof. Let $s, r_{0}>0$ and $B_{r_{0}}:=\left\{(x, y) \in \mathbb{R}^{d} \times \mathbb{R}^{d}\right.$ : $\left.|(x, y)|<r_{0}\right\}$, where $|(x, y)|=\left(|x|^{2}+|y|^{2}\right)^{1 / 2}$. Fix $r_{0}$ such that $\mu_{k} \otimes \mu_{k}\left(B_{r_{0}}\right)<1$. We write

$$
\begin{aligned}
\left\|V_{g}(f)\right\|_{L^{2}\left(\mu_{k} \otimes \mu_{k}\right)}^{2}= & \left\|\chi_{B_{r_{0}}} V_{g}(f)\right\|_{L^{2}\left(\mu_{k} \otimes \mu_{k}\right)}^{2} \\
& +\left\|\chi_{\mathbb{R}^{d} \backslash B_{r_{0}}} V_{g}(f)\right\|_{L^{2}\left(\mu_{k} \otimes \mu_{k}\right)}^{2} \\
\leq & \left\|\chi_{B_{r_{0}}} V_{g}(f)\right\|_{L^{2}\left(\mu_{k} \otimes \mu_{k}\right)}^{2} \\
& +r_{0}^{-2 s}\left\||(x, y)|^{s} V_{g}(f)\right\|_{L^{2}\left(\mu_{k} \otimes \mu_{k}\right)}^{2} .
\end{aligned}
$$

But from Hölder's inequality and Proposition 4(iii) we have

$$
\begin{aligned}
& \left\|\chi_{B_{r_{0}}} V_{g}(f)\right\|_{L^{2}\left(\mu_{k} \otimes \mu_{k}\right)}^{2} \\
& \quad \leq \mu_{k} \otimes \mu_{k}\left(B_{r_{0}}\right)\left\|V_{g}(f)\right\|_{L^{\infty}\left(\mu_{k} \otimes \mu_{k}\right)}^{2} \\
& \quad \leq \mu_{k} \otimes \mu_{k}\left(B_{r_{0}}\right)\|f\|_{L^{2}\left(\mu_{k}\right)}^{2}\|g\|_{L_{\text {rad }}^{2}\left(\mu_{k}\right)}^{2} .
\end{aligned}
$$


Therefore, by Theorem 5(i),

$$
\begin{aligned}
& r_{0}^{2 s}\left(1-\mu_{k} \otimes \mu_{k}\left(B_{r_{0}}\right)\right)\|f\|_{L^{2}\left(\mu_{k}\right)}^{2}\|g\|_{L_{\mathrm{rad}}^{2}\left(\mu_{k}\right)}^{2} \\
& \quad \leq\left\||(x, y)|^{s} V_{g}(f)\right\|_{L^{2}\left(\mu_{k} \otimes \mu_{k}\right)}^{2} .
\end{aligned}
$$

Using the fact that $|(x, y)|^{s}=\left(|x|^{2}+|y|^{2}\right)^{s / 2} \leq 2^{s / 2}\left(|x|^{s}+|y|^{s}\right)$ we deduce that

$$
\begin{aligned}
& 2 c(k, s)\|f\|_{L^{2}\left(\mu_{k}\right)}^{2}\|g\|_{L_{\mathrm{rad}}^{2}\left(\mu_{k}\right)}^{2} \\
& \quad \leq\left\||x|^{s} V_{g}(f)\right\|_{L^{2}\left(\mu_{k} \otimes \mu_{k}\right)}^{2}+\left\||y|^{s} V_{g}(f)\right\|_{L^{2}\left(\mu_{k} \otimes \mu_{k}\right)}^{2},
\end{aligned}
$$

where

$$
c(k, s)=\frac{r_{0}^{2 s}}{2^{s+1}}\left(1-\mu_{k} \otimes \mu_{k}\left(B_{r_{0}}\right)\right) .
$$

Replacing $f$ and $g$ by $f_{\lambda}$ and $g_{\lambda}$, respectively, in the previous inequality, we obtain by Lemma 10 and by a suitable change of variables

$$
\begin{aligned}
2 c(k, s) & \|f\|_{L^{2}\left(\mu_{k}\right)}^{2}\|g\|_{L_{\mathrm{rad}}^{2}\left(\mu_{k}\right)}^{2} \\
\leq & \lambda^{2 s}\left\||x|^{s} V_{g}(f)\right\|_{L^{2}\left(\mu_{k} \otimes \mu_{k}\right)}^{2} \\
& +\lambda^{-2 s}\left\||y|^{s} V_{g}(f)\right\|_{L^{2}\left(\mu_{k} \otimes \mu_{k}\right)}^{2} .
\end{aligned}
$$

By setting $\lambda=\left(\left\||y|^{s} V_{g}(f)\right\|_{L^{2}\left(\mu_{k} \otimes \mu_{k}\right)} /\left\||x|^{s} V_{g}(f)\right\|_{L^{2}\left(\mu_{k} \otimes \mu_{k}\right)}\right)^{1 / 2 s}$ in the right-hand side of the previous inequality we obtain the desired result.

We will now prove a local uncertainty principle for the Dunkl-Wigner transform $V_{g}$, which extends the result of Faris [22].

Theorem 12 (local uncertainty principle for $V_{g}$ ). Let $s>0$. Then there exists a constant $b(k, s)>0$ such that, for all $f \in$ $L^{2}\left(\mu_{k}\right)$ and $g \in L_{\text {rad }}^{2}\left(\mu_{k}\right)$ and for all measurable subset $E$ of $\mathbb{R}^{d} \times \mathbb{R}^{d}$ such that $0<\mu_{k} \otimes \mu_{k}(E)<\infty$, one has

$$
\begin{aligned}
& \left\|\chi_{E} V_{g}(f)\right\|_{L^{2}\left(\mu_{k} \otimes \mu_{k}\right)} \\
& \quad \leq b(k, s)\left(\mu_{k} \otimes \mu_{k}(E)\right)^{1 / 2}\left\||(x, y)|^{s} V_{g}(f)\right\|_{L^{2}\left(\mu_{k} \otimes \mu_{k}\right)} .
\end{aligned}
$$

Proof. Let $s>0$ and let $E$ be a measurable subset of $\mathbb{R}^{d} \times \mathbb{R}^{d}$ such that $0<\mu_{k} \otimes \mu_{k}(E)<\infty$. From Hölder's inequality and Proposition 4(iii) we have

$$
\begin{aligned}
& \left\|\chi_{E} V_{g}(f)\right\|_{L^{2}\left(\mu_{k} \otimes \mu_{k}\right)} \\
& \quad \leq\left(\mu_{k} \otimes \mu_{k}(E)\right)^{1 / 2}\left\|V_{g}(f)\right\|_{L^{\infty}\left(\mu_{k} \otimes \mu_{k}\right)} \\
& \quad \leq\left(\mu_{k} \otimes \mu_{k}(E)\right)^{1 / 2}\|f\|_{L^{2}\left(\mu_{k}\right)}\|g\|_{L_{\text {rad }}^{2}\left(\mu_{k}\right)} .
\end{aligned}
$$

From (63) there exists $b(k, s)>0$ such that

$$
\begin{aligned}
& \|f\|_{L^{2}\left(\mu_{k}\right)}\|g\|_{L_{\mathrm{rad}}^{2}\left(\mu_{k}\right)} \\
& \quad \leq\left. b(k, s)\|\|(x, y)\right|^{s} V_{g}(f) \|_{L^{2}\left(\mu_{k} \otimes \mu_{k}\right)} .
\end{aligned}
$$

Therefore we obtain the desired result.

\section{Competing Interests}

The author declares that there is no conflict of interests regarding the publication of this paper.

\section{References}

[1] C. F. Dunkl, "Hankel transforms associated to finite reection groups," Contemporary Mathematics, vol. 138, pp. 123-138, 1992.

[2] M. Rösler, "An uncertainty principle for the Dunkl transform," Bulletin of the Australian Mathematical Society, vol. 59, no. 3, pp. 353-360, 1999.

[3] N. Shimeno, "A note on the uncertainty principle for the Dunkl transform," The University of Tokyo. Journal of Mathematical Sciences, vol. 8, no. 1, pp. 33-42, 2001.

[4] F. Soltani, "Heisenberg-Pauli-Weyl uncertainty inequality for the Dunkl transform on $\mathbb{R}^{d, " B u l l e t i n}$ of the Australian Mathematical Society, vol. 87, no. 2, pp. 316-325, 2013.

[5] F. Soltani, "A general form of Heisenberg-Pauli-Weyl uncertainty inequality for the Dunkl transform," Integral Transforms and Special Functions, vol. 24, no. 5, pp. 401-409, 2013.

[6] F. Soltani, "An $L^{p}$ Heisenberg-Pauli-Weyl uncertainty principle for the Dunkl transform," Konuralp Journal of Mathematics, vol. 2, no. 1, pp. 1-6, 2014.

[7] F. Soltani, "Uncertainty principles and extremal functions for the Dunkl $L^{2}$-multiplier operators," Journal of Operators, vol. 2014, Article ID 659069, 9 pages, 2014.

[8] S. Thangavelu and Y. Xu, "Convolution operator and maximal function for the Dunkl transform," Journal d'Analyse Mathématique, vol. 97, pp. 25-55, 2005.

[9] F. Soltani, "Inversion formula for the Dunkl-Wigner transform and compactness property for the Dunkl-Weyl transforms," Journal of Mathematical Research with Applications, vol. 35, no. 4, pp. 425-434, 2015.

[10] F. Soltani, "Reproducing inversion formulas for the DunklWigner transforms," Cubo: A Mathematical Journal, vol. 17, no. 2, pp. 1-14, 2015.

[11] H. Weyl, The Theory of Groups and Quantum Mechanics, Dover, New York, NY, USA, 1950.

[12] M. W. Wong, Weyl Transforms, Universitext, Springer, New York, NY, USA, 1998.

[13] A. Dachraoui, "Weyl-Bessel transforms," Journal of Computational and Applied Mathematics, vol. 133, no. 1-2, pp. 263-276, 2001.

[14] C. F. Dunkl, "Integral kernels with reflection group invariance," Canadian Journal of Mathematics, vol. 43, no. 6, pp. 1213-1227, 1991.

[15] M. F. E. de Jeu, “The Dunkl transform,” Inventiones Mathematicae, vol. 113, no. 1, pp. 147-162, 1993.

[16] E. M. Opdam, "Dunkl operators, Bessel functions and the discriminant of a finite Coxeter group," Compositio Mathematica, vol. 85 , no. 3, pp. 333-373, 1993.

[17] M. Rösler, "Positivity of Dunkl's intertwining operator," Duke Mathematical Journal, vol. 98, no. 3, pp. 445-463, 1999.

[18] M. Rösler and M. Voit, "Markov processes related with Dunkl operators," Advances in Applied Mathematics, vol. 21, no. 4, pp. 575-643, 1998.

[19] G. N. Watson, A Treatise on Theory of Bessel Functions, Cambridge University Press, Cambridge, UK, 1966. 
[20] M. Rösler, "A positive radial product formula for the Dunkl kernel," Transactions of the American Mathematical Society, vol. 355, no. 6, pp. 2413-2438, 2003.

[21] S. Thangavelu and Y. Xu, "Riesz transform and Riesz potentials for Dunkl transform," Journal of Computational and Applied Mathematics, vol. 199, no. 1, pp. 181-195, 2007.

[22] W. G. Faris, "Inequalities and uncertainty inequalities," Journal of Mathematical Physics, vol. 19, pp. 461-466, 1978. 


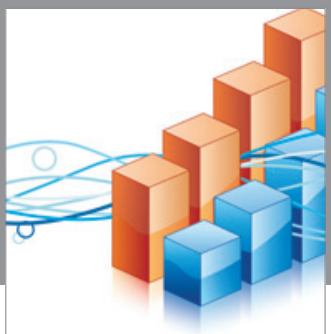

Advances in

Operations Research

vatem alat4

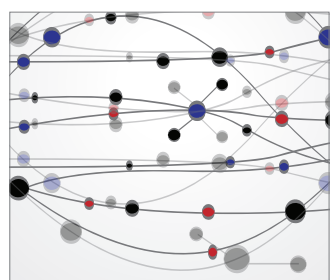

\section{The Scientific} World Journal
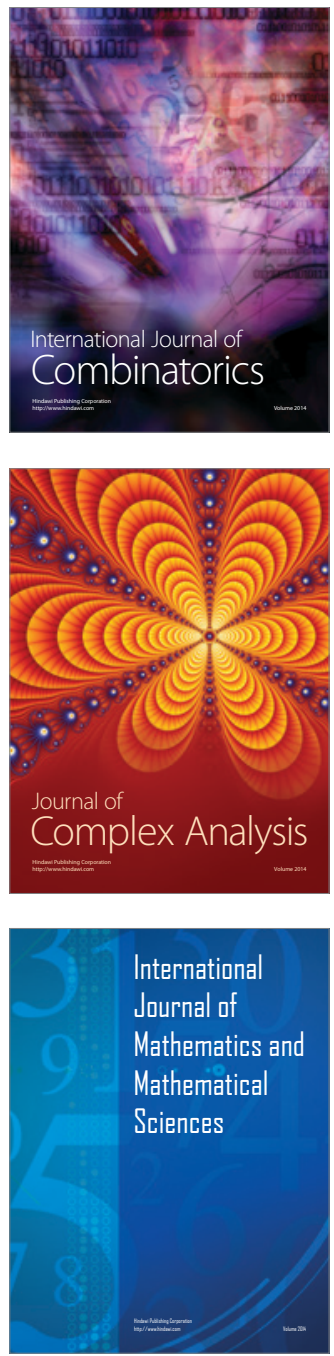
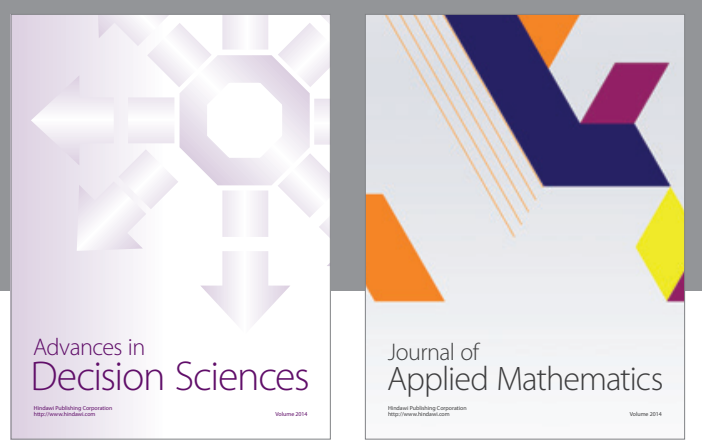

Algebra

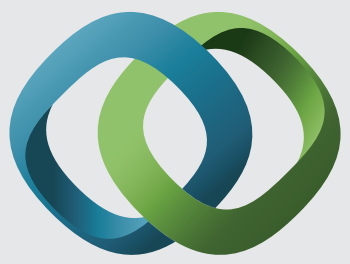

\section{Hindawi}

Submit your manuscripts at

http://www.hindawi.com
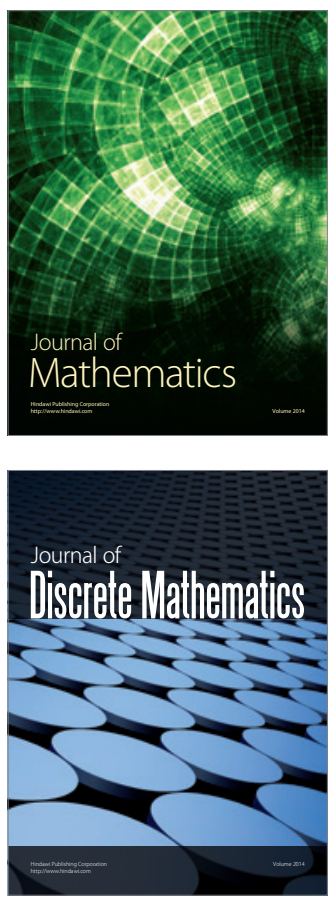

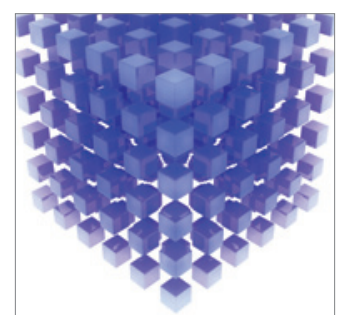

Mathematical Problems in Engineering
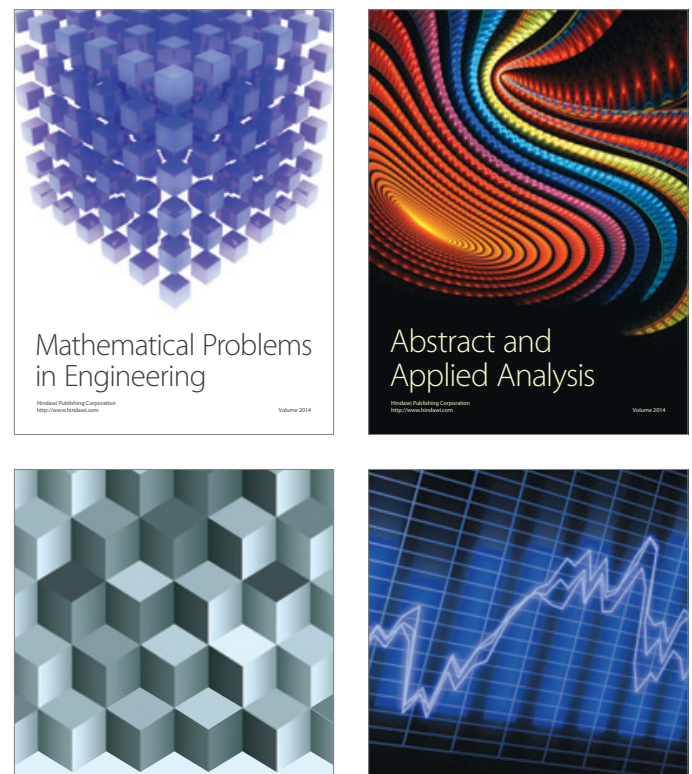

Journal of

Function Spaces

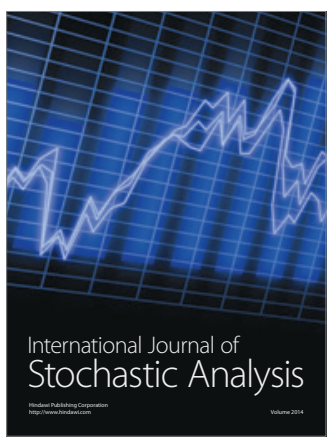

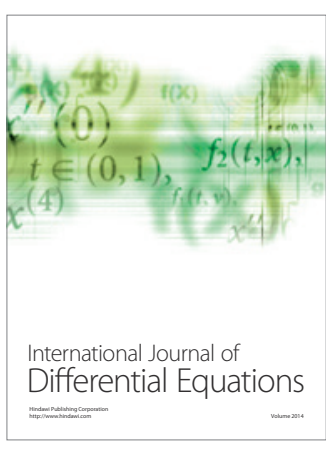
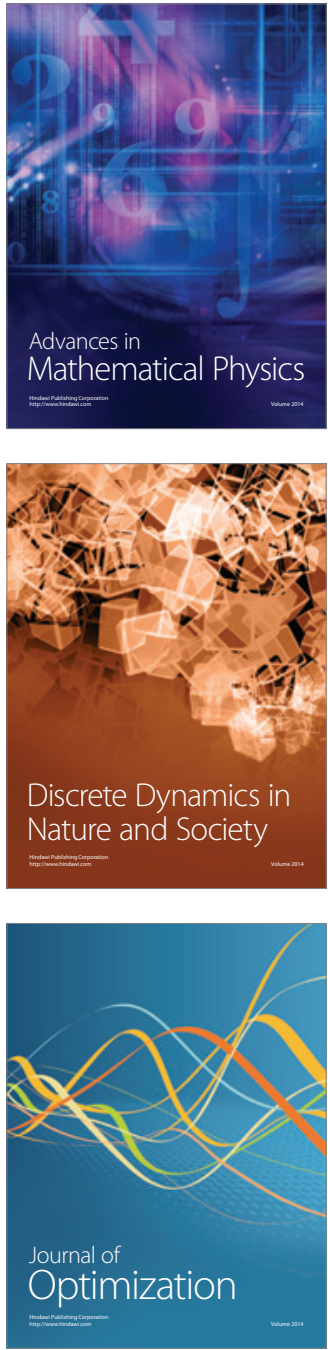